University of Wollongong

Research Online

Australian Institute for Innovative Materials -

Papers

Australian Institute for Innovative Materials

$1-1-2016$

Fabrication and characterisation of GaAs nanopillars using nanosphere lithography and metal assisted chemical etching

A Cowley

European Space Agency, Dublin City University

J A. Steele

University of Wollongong

D Byrne

Dublin City University

R K. Vijayaraghavan

Dublin City University

P J. McNally

Dublin City University

Follow this and additional works at: https://ro.uow.edu.au/aiimpapers

Part of the Engineering Commons, and the Physical Sciences and Mathematics Commons

Research Online is the open access institutional repository for the University of Wollongong. For further information contact the UOW Library: research-pubs@uow.edu.au 


\title{
Fabrication and characterisation of GaAs nanopillars using nanosphere lithography and metal assisted chemical etching
}

\author{
Abstract \\ We present a low-cost fabrication procedure for the production of nanoscale periodic GaAs nanopillar \\ arrays, using the nanosphere lithography technique as a templating mechanism and the electrochemical \\ metal assisted etch process (MacEtch). The room-temperature photoluminescence (PL) and Raman \\ spectroscopic properties of the fabricated pillars are detailed, as are the structural properties (scanning \\ electron microscopy) and fabrication process. From our PL measurements, we observe a singular GaAs \\ emission at $1.43 \mathrm{eV}$ with no indications of any blue or green emissions, but with a slight redshift due to \\ porosity induced by the MacEtch process and characteristic of porous GaAs ( $p-G a A s)$. This is further \\ confirmed via Raman spectroscopy, where additionally we observe the formation of an external cladding \\ of elemental As around our nanopillar features. The optical emission is enhanced by an order magnitude \\ $(\sim 300 \%)$ for our nanopillar sample relative to the planar unprocessed GaAs reference.

\section{Keywords} \\ nanopillars, gaas, metal, lithography, characterisation, assisted, etching, fabrication, nanosphere, chemical

\section{Disciplines} \\ Engineering | Physical Sciences and Mathematics

\section{Publication Details} \\ Cowley, A., Steele, J. A., Byrne, D., Vijayaraghavan, R. K. \& McNally, P. J. (2016). Fabrication and \\ characterisation of GaAs nanopillars using nanosphere lithography and metal assisted chemical etching. \\ RSC Advances: an international journal to further the chemical sciences, 6 30468-30473.
}




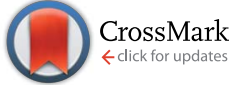

Cite this: RSC Adv., 2016, 6, 30468

Received 9th November 2015

Accepted 14th March 2016

DOI: 10.1039/c5ra23621e

www.rsc.org/advances

\section{Fabrication and characterisation of GaAs nanopillars using nanosphere lithography and metal assisted chemical etching}

\author{
A. Cowley, ${ }^{\text {ad }}$ J. A. Steele, ${ }^{\text {b D. Byrne, }}{ }^{\text {C R. K. Vijayaraghavana and P. J. McNally }}{ }^{a}$
}

We present a low-cost fabrication procedure for the production of nanoscale periodic GaAs nanopillar arrays, using the nanosphere lithography technique as a templating mechanism and the electrochemical metal assisted etch process (MacEtch). The room-temperature photoluminescence (PL) and Raman spectroscopic properties of the fabricated pillars are detailed, as are the structural properties (scanning electron microscopy) and fabrication process. From our PL measurements, we observe a singular GaAs emission at $1.43 \mathrm{eV}$ with no indications of any blue or green emissions, but with a slight redshift due to porosity induced by the MacEtch process and characteristic of porous GaAs ( $\pi$-GaAs). This is further confirmed via Raman spectroscopy, where additionally we observe the formation of an external cladding of elemental As around our nanopillar features. The optical emission is enhanced by an order magnitude ( 300\%) for our nanopillar sample relative to the planar unprocessed GaAs reference.

\section{Introduction}

III-V compound semiconductor nanostructures have been shown to be promising materials for a variety of optoelectronic and energy related applications such as light emitting diodes (LEDs), photovoltaics (PV) and field effect transistors (FETs). Such nanostructures can be formed either using top-down approaches, such as reactive ion etching (RIE) or bottom-up growth methods. Understanding the processing limitations and advantages/disadvantages of alternate nanostructure fabrication approaches is important for progressing this field, and is the motivation for this work.

MacEtch is a relatively simple wet etch technique that allows for the fabrication of nanostructures, and has been widely applied in recent years to silicon-based technologies. ${ }^{1-3}$ Developing this approach for III-V and other compound semiconductors remains challenging, owing to the stoichiometric difficulties of reactions with two or more elements, however it is attractive owing to its low-cost, fast and non-thermal character relative to other III-V nanostructure fabrication approaches.

Fundamentally, the MacEtch approach requires the effective masking of a crystalline semiconductor material with a noble metal layer ( $\mathrm{Au}, \mathrm{Ag}$ and $\mathrm{Pt}$ are commonly reported metals for this approach $\left.^{\mathbf{1 - 4}}\right)$. This metal layer acts as a catalyst for the controlled

\footnotetext{
${ }^{a}$ Nanomaterials Processing Laboratory, School of Electronic Engineering, Dublin City University, Dublin 9, Ireland. E-mail: cougaris@gmail.com

${ }^{b}$ Institute for Superconducting and Electronic Materials, School of Physics, University of Wollongong, Wollongong, New South Wales 2522, Australia

'School of Physical Sciences, Dublin City University, Dublin 9, Ireland

${ }^{d}$ European Space Agency, Linder Hohe, D-51147 Cologne, Germany
}

etch process, wherein hole carriers $\left(\mathrm{h}^{+}\right)$are transferred through the catalyst to the underlying crystalline semiconductor, resulting in oxidation at the metal-semiconductor interface. An oxide removing acid (e.g. hydrofluoric acid, HF) can then result in the removal of the oxide layer produced thusly, allowing for controlled development of etch features (i.e., a nanopillar). As an analogy, the metal mask essentially sinks into the semiconductor substrate and the unmasked regions are 'extruded'. This oxide formation via oxidizer at the interface and removal via acid etch must be maintained throughout the process, and thus MacEtch solutions are comprised of both oxidant and acid. For $\mathrm{Si}$, classically a solution of $\mathrm{HF}$ and $\mathrm{H}_{2} \mathrm{O}_{2}$ is used for this process with, typically, an Au metal mask.

Nanostructure formation using the MacEtch approach has been carried out before for III-V materials. Micro n-type convex GaAs arrays were created using Pt/Pd catalyst metal pattern combined with a nanosphere mask, employing an etchant composed of $\mathrm{HF} / \mathrm{H}_{2} \mathrm{O}_{2} .{ }^{5}$ Periodic 'microbump' arrays of InP have been created using a similar etchant mixture driven by UV photoirradiation, however the size of the structures fabricated were in the order of microns. ${ }^{6}$ For GaAs, a different oxidizer is required, as $\mathrm{H}_{2} \mathrm{O}_{2}$ will isotropically etch GaAs unassisted. The experiments of DeJarld et al. ${ }^{7}$ detail an alternative approach for GaAs MacEtch, using a weaker oxidizing agent $\left(\mathrm{KMnO}_{4}\right)$. They were able to successfully create high aspect ratio $600 \mathrm{~nm}$-wide GaAs nanopillars using an $\mathrm{Au}$ mask and $\mathrm{H}_{2} \mathrm{SO}_{4} / \mathrm{KMnO}_{4}$ solution, at an etch temperature of roughly $40-45{ }^{\circ} \mathrm{C}$.

In this paper, we outline the simple, low-cost approach using a polystyrene (PS) nanosphere mask to fabricate GaAs nanopillar structures, and report on the morphology, PL and Raman scattering properties of our structures. 


\section{Experimental}

Epi-ready n-type Si-doped GaAs wafers were supplied via University Wafer, Inc. Single 2" wafers of n-GaAs (100) substrate with carrier concentrations of approximately $1.5-2.8 \times 10^{18} \mathrm{~cm}^{3}$ (resistivity in the order of $1.1 \times 10^{-3} \Omega \mathrm{cm}$ ) were used as the bulk templates. Prior to nanopillar formation experiments, the GaAs substrates were partitioned into $1 \mathrm{~cm}^{2}$ pieces.

Previous nanosphere lithography patterning processes fundamentally inform our experimental approach. ${ }^{8,9} \mathrm{~A}$ schematic of the general stepwise fabrication process is given in Fig. 1. Polystyrene (PS) nanospheres of $1 \mu \mathrm{m}$ diameter (std dev. < $0.1 \mu \mathrm{m}$ ) were used, supplied by Sigma Aldrich. Prior to the application of PS nanospheres, our GaAs coupons were subjected to native oxide removal using dilute $\mathrm{HCl}$. In order to improve the GaAs etch interface and turn the GaAs surface hydrophilic, we exposed it to a $\mathrm{NH}_{4} \mathrm{OH}: \mathrm{H}_{2} \mathrm{O}_{2}: \mathrm{H}_{2} \mathrm{O}$ solution $(1: 1: 10)$.

PS nanosphere templates were prepared on the surface of water by diluting a $10 \%$ wt solution of $\sim 1 \mu \mathrm{m}$ PS nanospheres with an equal volume of ethanol. The diluted solution was then dispersed onto the surface of water and the nanospheres allowed to assemble in an ordered close-packed monolayer crystal. A uniform nanosphere template was transferred onto the GaAs substrates by submerging the coupons and lifting them through the nanosphere monolayer. Once coated, our samples were allowed to dry. For size reduction of the PS nanosphere mask, an Oxford Instruments PlasmaLab RIE 2000 was used, for an etch time of 75 seconds. Variation of the etch time can lead to differing nanopillar diameters, however for this work a set diameter of approximately $600 \mathrm{~nm}$ was desired. PS size reduction was carried out with an $\mathrm{O}_{2}$ plasma $(50 \mathrm{sccm})$, operating at $300 \mathrm{~W}$ RF power at 100 mtorr chamber pressure. After plasma etching, $20 \mathrm{~nm}$ of gold (99.99\%, Sigma Aldrich)

(1)

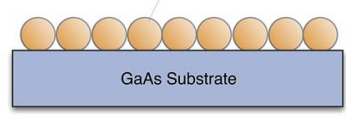

(3)

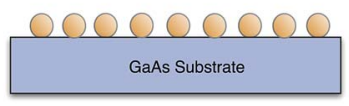

(5) Au layer $\sim 20 \mathrm{~nm}$

GaAs Substrate

Fig. 1 Illustrative schematic of the process flow leading to the formation of the GaAs nanopillars. (1) Polystyrene (PS) nanospheres deposited onto the GaAs substrate, (2\&3) size reduction of the PS nanosphere mask via reactive-ion etching (RIE), (4) deposition of catalyst Au metal via PVD, (5) removal of PS nanospheres, leaving the Au mask intact, (6) chemical etching of GaAs using metal catalyst and formation of nanopillar structure. was evaporated on top of the substrates using vacuum evaporation physical vapor deposition (PVD) at a rate of $0.1 \mathrm{~nm} \mathrm{~s}^{-1}$.

For the etch procedure, the gold-templated substrates were first submerged in a small amount of ethanol to wet the surface. The separately prepared MacEtch solution of $20 \mathrm{ml} \mathrm{HF}, 20 \mathrm{ml}$ ethanol and $0.03 \mathrm{~g} \mathrm{KMnO}_{4}$ was then added. After the samples were submerged in the final MacEtch solution for a time of 4 to $7 \mathrm{~min}$, they were removed, washed with ethanol, and dried prior to subsequent characterization.

For detailed investigation of the nanopillars, roomtemperature Raman spectra were acquired in an unpolarised quasi-backscattering configuration on the (100) GaAs sample surfaces, using a Jobin-Yvon HR800 micro-Raman system, $\sim 10 \mathrm{~mW} 488 \mathrm{~nm}$ laser excitation $\left(\mathrm{Ar}^{+}\right.$source), and an air-cooled CCD detector. Dispersion was achieved using an $1800 \mathrm{~g} \mathrm{~mm}^{-1}$ grating and spectra were recorded with a spectral resolution of $0.2 \mathrm{~cm}^{-1}$, with a spatial resolution of $\sim 2 \mu \mathrm{m}$. The same system was also used for the room-temperature PL measurements, using $325 \mathrm{~nm}$ UV He-Cd laser which was focused to a diameter of $\sim 2 \mu \mathrm{m}$ on the sample surface using a UV objective lens. Scanning electron microscopy (SEM) images were taken of the samples using a Karl Zeiss EVO.

\section{Results \& discussion}

Fig. 2 presents SEM micrographs of two GaAs nanopillar structures produced using our MacEtch procedure, with samples submerged in the etching solution for 4 and $7 \mathrm{~min}$. From the experimental conditions outlined above, we can estimate the room-temperature vertical etch rate to be approximately $150 \mathrm{~nm} \mathrm{~min}{ }^{-1}$ MacEtch proceeds with the production of holes by the metal catalyst and oxidation of the GaAs with these holes. Removal of the oxidized and $\pi$-GaAs is carried out by the HF etchant. Some lateral etch is also evident, giving rise to the rounded top profile. This is a parameter that could potentially be improved by greater control of the etch solution, i.e. by decreasing oxidant and acid concentrations.

Variations in the individual morphology of the GaAs nanopillars can be clearly seen, as can a slight height difference across the sample. The height variation is likely caused by etch rate variation across the Au catalyst layer, and may be attributed to the quality of the $\mathrm{Au} / \mathrm{GaAs}$ interface. In earlier experiments, directly immersing the Au templated coupon into the MacEtch solution would sometimes lead to delamination of the metal catalyst layer. With improvements to the experimental method, primarily by pre-wetting the GaAs coupon prior to immersion, the delamination problem was overcome. This observation still hints at the potential issue of the $\mathrm{Au} / \mathrm{GaAs}$ interface that may be responsible for this local variation. Another possibility is localized etch solution concentration gradients. It may be that using an approach for forming n-type GaAs ohmic contacts with $\mathrm{Au} / \mathrm{Ge}$ or $\mathrm{Au} / \mathrm{Ge} / \mathrm{Ni}$ alloy, instead of pure $\mathrm{Au}$, could also improve the catalyst layer adhesion and conduction properties.

The circular shaped pillars have diameters of approximately $600 \mathrm{~nm}$, matching the Au pattern mask hole diameter, with vertical heights in the range of $0.5-1.2 \mu \mathrm{m}$, depending on etch time, and with an aspect ratio of $2: 1$ at the vertical maximum observed. 
(A)

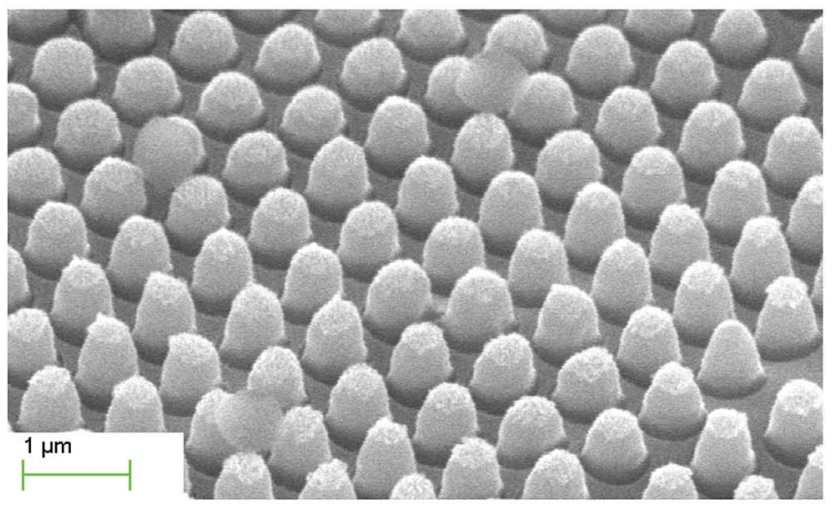

(B)

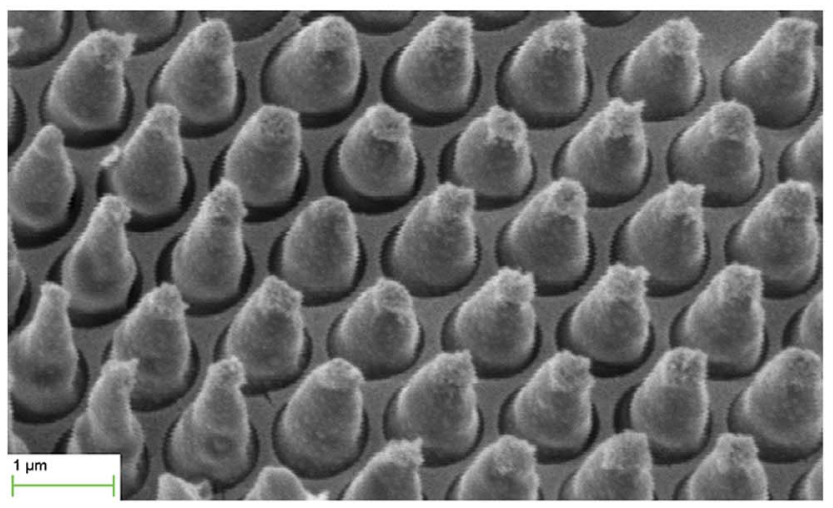

Fig. 2 SEM images taken at $45^{\circ}$ to the surface normal revealing the highly-ordered nanopillar morphology of our GaAs samples etched for a duration of (A) 4 min and (B) 7 min.

\section{Photoluminescence}

In Fig. 3, we display the room-temperature PL spectra acquired from the nanostructured GaAs surface as well as the unprocessed c-GaAs coupon, for reference. The primary emission for the GaAs nanopillar sample is observed to peak at $1.43 \mathrm{eV}$. The PL spectrum of the c-GaAs control is characterized by the presence of one sharp emission band in the region of fundamental bandgap of GaAs $\left(E_{g}=1.424 \mathrm{eV}\right)$, with the peak centered at $864 \mathrm{~nm}$. Relative to the reference GaAs sample, the PL peak wavelength recorded from the GaAs nanopillars is redshifted by approximately $3 \mathrm{~nm}$.

This change in optical properties is well-reported for $\pi$-GaAsrelated materials and is not unexpected. ${ }^{10-12}$ It is explained either as the variation of the $\pi$-GaAs lattice parameter (and subsequent variation of the strain-state),${ }^{10}$ to that of c-GaAs, or by localized perturbations of the conduction and valence band edges caused by the layer porosity. ${ }^{12}$ From our measurements, no other emissions are observed. This is also interesting, as again in previous studies of $\pi$-GaAs, high-energy green and blue emissions ( $\sim 440 \mathrm{~nm}$ and $\sim 560 \mathrm{~nm}$ respectively) are frequently reported. These emissions are well attributed to quantumconfined excitonic luminescence, due to small GaAs nanocrystals embedded in the $\pi$-GaAs layer or defects within the $\pi$-GaAs oxide layer. The measured PL emission intensity is

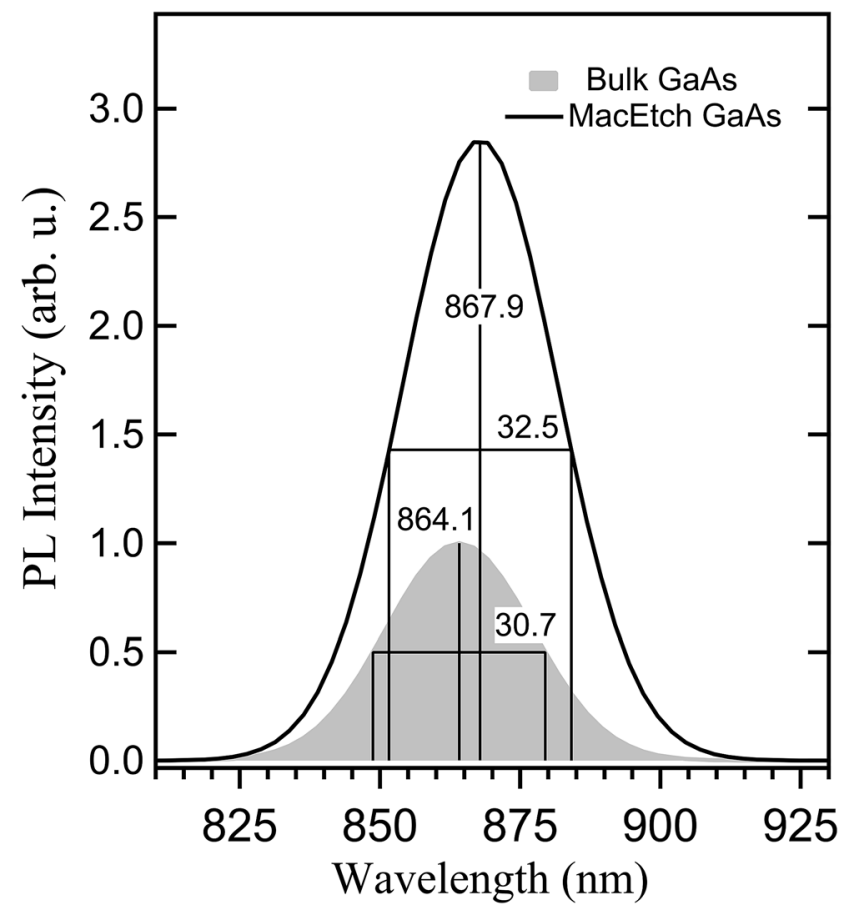

Fig. 3 Room-temperature PL spectra obtained from MacEtch GaAs nanopillars (full width half maximum $32.5 \mathrm{~nm}$ ) and a baseline unprocessed GaAs substrate (full width half maximum $30.7 \mathrm{~nm}$ ).

notably enhanced by approximately $300 \%$ for the nanopillar samples, relative to our c-GaAs reference. The emission of light from the planar, untextured c-GaAs surface has a limited, narrow escape cone and is prone to internal reflection and reabsorption. ${ }^{13}$ Nanopillar type structures increase the surface area directly available for luminescence and reduce radiative loses, resulting in a marked improvement in the intensity.

\section{Raman spectroscopy}

Chemical changes occurring due to etching the GaAs surface can readily be investigated using methods such as energydispersive X-ray spectroscopy (EDS). However, the more subtle effects of product aggregation, oxide formations, and lattice defects, must be determined using a phase specific technique such as Raman spectroscopy. An anticipated consequence of the MacEtch process is the formation of a new surrounding medium, which clads the GaAs core (see inset of Fig. 4), and which is composed of by-products from the etching reaction.

Detailed studies of subsurface solid/solid interfaces are particularly challenging and are important to the MacEtch technique validity. Within the context of the present study, and its potential for device application, the chemical reactions occurring at the GaAs interface must be well understood as it may contribute to the development of fundamental phenomena such as Schottky barrier formation or Fermi level pinning.

For the case of the GaAs nanopillars, Raman spectra recorded from several surface locations revealed that the resultant MacEtch-induced changes (surface morphology and chemistry) were highly homogenous with respect to phonon frequencies 


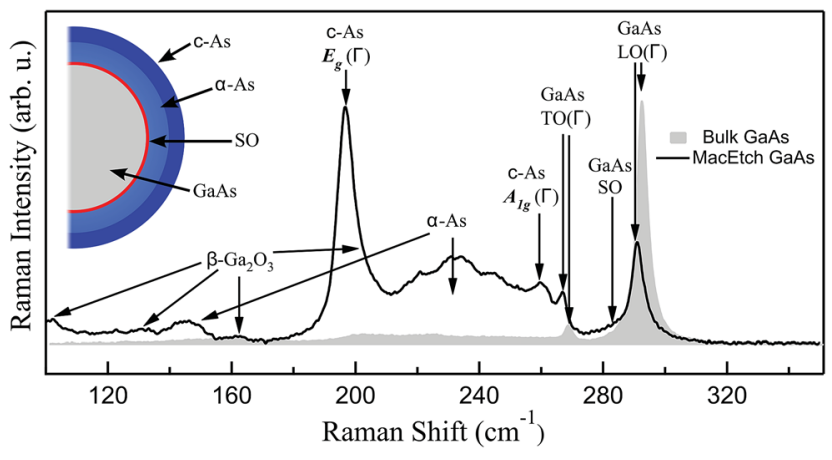

Fig. 4 Normalized room-temperature Raman spectra recorded from bulk and MacEtch (100) GaAs surfaces, with the labelled arrows indicating the origins of spectral features. The inset shows a schematic of the proposed nanopillar cross-section, as interpreted from the Raman data and the basic thermodynamics of the $\mathrm{Ga}$-As-O phase diagram. ${ }^{25}$

and general Raman lineshapes. Fig. 4 presents typical roomtemperature micro-Raman spectra recorded from the (100) GaAs nanopillar surface and bulk (100) GaAs, along with a schematic of the formed nanopillar cross-section displayed in the inset. An initial comparison of these two spectra reveals significant differences in the spectral range of $180-270 \mathrm{~cm}^{-1}$, where the $\mathrm{E}_{g}\left(196 \mathrm{~cm}^{-1}\right)$ and $\mathrm{A}_{1 \mathrm{~g}}\left(257 \mathrm{~cm}^{-1}\right)$ modes of crystalline arsenic (c-As) are known to occur ${ }^{14}$ and where the amorphous arsenic $\left(\alpha-\right.$ As) exhibits a broadband feature $\left(180-270 \mathrm{~cm}^{-1}\right)$ and weaker low frequency peak $\left(146 \mathrm{~cm}^{-1}\right) \cdot{ }^{15}$ The anomalously large intensity of the c-As $E_{g}$ mode, relative to $A_{1 g}$ mode observed here, is associated with a low temperature reaction and can be understood by an additional contribution from a peak of approximately the same frequency originating in $\alpha$-As. ${ }^{16}$ This does not imply that a well-defined simple molecular unit exists within the $\alpha$-As, however it may form in an arsenic system that is undergoing a phase transition: $\alpha$-As $\rightarrow$ c-As.

A transitioning arsenic system may also aid in the interpretation of the $\alpha$-As broadband contour observed in Fig. 4, and the superposition of smaller peaks which is more representative for comparison with the results of other Raman studies performed on $\pi$-GaAs - achieved through an oxidation etching procedure ${ }^{17-19}$ - rather than the published spectra of $\alpha$-As. ${ }^{15}$ For completeness, we point out that none of the peak frequencies of these vibrations align with any of the vibrations suggested for the various arsenic or gallium oxide polymorphs. ${ }^{20-23}$ As will become clear in later discussion, a distinct absence of $\mathrm{As}_{2} \mathrm{O}_{3}$ Raman signatures ${ }^{23,24}$ indicates its utility in driving the MacEtch process and the GaAs nanopillar surface chemistry to ultimate system equilibrium.

According to the Raman selection rules for a zincblende crystal ( $T_{\mathrm{d}}$ site symmetry), the $\mathrm{LO}(\Gamma)$ phonon is allowed for light backscattered from the (100) GaAs surface while $\mathrm{TO}(\Gamma)$ is forbidden. Consequently, the weak scattering of the TO modes from bulk (100) GaAs (see Fig. 3) can be interpreted by minor deviations from a pristine GaAs system which relax the selection rules. However the appearance of the symmetry-forbidden TO mode as a more prominent feature in the $\pi$-GaAs Raman spectra - when compared to the LO band - is attributed to lateral reflections off the tapered pillar sides and the subsequent loss of the initial scattering geometry.

\section{GaAs optical phonon redshift}

The GaAs TO and LO phonons are observed to broaden and redshift to lower frequencies in the GaAs nanopillar surface. The scattering volume of our nanopillars are not small enough to warrant the often-used spatial correlation interpretation, developed by Campbell and Fauchet. ${ }^{25}$ In addition, the formation of GaAs nanocrystallites is not believed to arise near the surface of our sample, as evidenced by an absence of the related green and blue emissions in our PL measurements. Evoking the concept of "phonon confinement", the redshift in the optical phonons most likely arise due to crystal defects introduced by the MacEtch processes. Softening occurs because the phonon, confined in direct space within a sphere of diameter $L$, can be described in reciprocal space by a wave packet with a range of $k$-values, $\Delta k \approx L-1 .^{26,27}$ While the lateral surface damage is likely shallow and uniform, it will be enough to soften the GaAs optical modes.

\section{Oxides}

During the experimental etch process the GaAs surface is dissolved according to the following reaction:

$$
\mathrm{GaAs}(\mathrm{s})+6 \mathrm{H}^{+} \rightarrow \mathrm{Ga}^{3+}(\mathrm{aq})+\mathrm{As}^{3+}(\mathrm{aq})
$$

The $\mathrm{Ga}^{3+}$ and $\mathrm{As}^{3+}$ is formed at the interface between the etch solution and the solid phase, while the dispersion of $\mathrm{Ga}_{2} \mathrm{O}_{3}$ and $\mathrm{As}_{2} \mathrm{O}_{3}$ oxides, as well as of GaAs particulates, will occur in the solution; thus, observations of Raman bands originating from the oxide products are expected.

The weaker peaks at $105 \mathrm{~cm}^{-1}, 134 \mathrm{~cm}^{-1}$ and $164 \mathrm{~cm}^{-1}$ are assigned to the formation of thermodynamically stable $\beta$-phase gallium trioxide $\left(\mathrm{Ga}_{2} \mathrm{O}_{3}\right)$, along with its largest contributing peak fortuitously superimposed on the high energy shoulder of the c-As $A_{g}$ phonon at $200 \mathrm{~cm}^{-1}$. We observe all $\beta-\mathrm{Ga}_{2} \mathrm{O}_{3}$ vibrations at frequencies slightly less than those reported for $\beta-\mathrm{Ga}_{2} \mathrm{O}_{3}$ single crystals, ${ }^{20}$ which suggests the formation of $\beta-\mathrm{Ga}_{2} \mathrm{O}_{3}$ nanostructures. ${ }^{21,22}$ The physical location of the gallium oxide, with respect to the schematic presented in the inset of Fig. 4, is difficult to deduce. It is likely to exist simply as loose residuals atop the surface rather than forming a well-defined deposit.

\section{Surface optical phonons (SO)}

The broad weaker peak on the low frequency side of the GaAs LO phonon is also clearly resolved. A detailed analysis of this peak, which will be presented in the following, allows us to assign it to scattering from surface optical (SO) phonons. While SO modes are relatively weak and generally require quite large surface-to-volume ratios to be resolved, we suggest the orientation of the nanopillars - relative to the Raman backscattering geometry - and the nanopillar height helps to enhance the relative strength held by this mode within the scattering volume. 
The SO mode dispersion at the interface between the GaAs nanopillar and a dielectric material can be calculated taking into account the geometrical constraint and the dielectric constant of the surrounding medium, $\varepsilon_{\mathrm{m}} \cdot{ }^{28}$ For a near cylindrical interface of radius $r$, the SO mode frequency is given by

$$
\omega_{\mathrm{SO}}^{2}=\omega_{\mathrm{TO}}^{2}+\frac{\tilde{\omega}_{\mathrm{p}}{ }^{2}}{\varepsilon_{\infty}+\varepsilon_{\mathrm{m}} f(q r)}
$$

where

$$
f(q r)=\frac{I_{0}(q r) K_{1}(q r)}{I_{1}(q r) K_{0}(q r)}
$$

here the screened phonon frequency $\tilde{\omega}_{\mathrm{p}}{ }^{2}$ is determined by $\omega_{\mathrm{LO}}{ }^{2}$ $=\omega_{\mathrm{TO}}{ }^{2}+\tilde{\omega}_{\mathrm{p}}{ }^{2} / \varepsilon_{\infty}$, with $I_{j}(q r)$ and $K_{j}(q r)$ being the $j$ th order modified Bessel functions, $q$ is the phonon wavevector, and $\varepsilon_{\infty}$ is the high frequency dielectric constant of GaAs.

A typical application of eqn (2) is used to investigate radial dependencies, allowing a reasonably accurate estimate of the average cylinder radius if $\varepsilon_{\mathrm{m}}$ is known. However, our nanopillars satisfy the asymptotic criterion $(r \gg 100 \mathrm{~nm})$; therefore we plot in Fig. 5 our measured SO peak frequency $\left(281.8 \mathrm{~cm}^{-1}\right)$ and the expected SO phonon dispersion for the $\alpha$-As/GaAs interface using eqn (2). A value of $\varepsilon_{\mathrm{m}}=11.1$ for $\alpha-\mathrm{As}^{29}$ is in excellent agreement with our asymptotic limit for large $r$ and supports a model where an amorphous phase of elemental arsenic aggregates at the GaAs surface, as shown in the inset of Fig. 4.

\section{Arsenic segregation}

The formation of elemental arsenic during the surface oxidation of GaAs is now well established ${ }^{\mathbf{1 8 , 2 5 , 3 0}}$ and has been assigned to an interfacial solid-state reaction between $\mathrm{As}_{2} \mathrm{O}_{3}$ in the etching solution and the GaAs crystal. The reaction is related to the basic thermodynamics of the Ga-As-O phase diagram ${ }^{25}$ by:

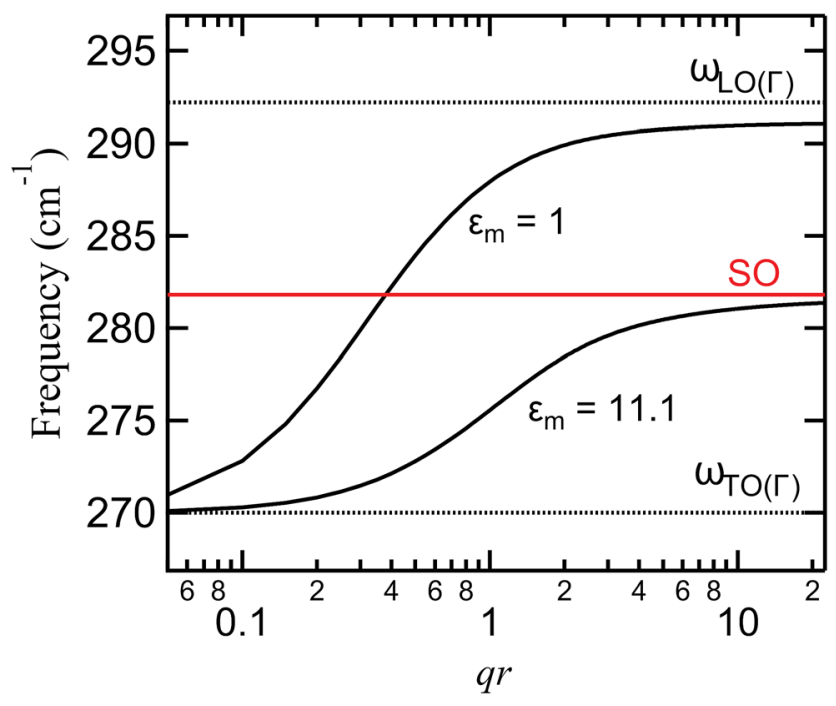

Fig. 5 Experimentally observed surface optical (SO) mode frequency and calculated dispersion of SO mode for GaAs interfacing with air $\left(\varepsilon_{m}=1\right)$ and $\alpha$-As $\left(\varepsilon_{m}=11.1\right) .{ }^{18}$ The two broken horizontal lines here represent the dispersionless longitudinal optical (LO) and transverse optical (TO) phonon frequencies.

$$
\mathrm{As}_{2} \mathrm{O}_{3}(\mathrm{~s})+2 \mathrm{GaAs}(\mathrm{s}) \rightarrow \mathrm{Ga}_{2} \mathrm{O}_{3}(\mathrm{~s})+4 \mathrm{As}(\mathrm{s})
$$

The utility of $\mathrm{As}_{2} \mathrm{O}_{3}$ reactants in this reaction makes clear its absence in the final GaAs nanopillar morphology and our measured Raman spectra. However the free As product cannot be overlooked; a sufficiently thick elemental arsenic deposit has formed to effectively absorb incident radiation. At a minimum, it is enough to significantly stifle optical abortion in the GaAs core and prevent possible photovoltaic applications. One might also note that c-As is a semimetal while $\alpha$-As is a narrow bandgap (1.1 eV) semiconductor.

Understanding the end arsenic morphology is important and, while it is outside the scope of the present study, it is challenging to predict or quantify. Work by Schwartz et al. ${ }^{18}$ on the detection sensitivity for arsenic inclusions in GaAs native oxide films suggests an upper limit of approximately $2 \mathrm{~nm}$ of As might go undetected. Moreover, since in a backscattering geometry both the excitation beam and the Raman scattered light are attenuated by the absorbing layer, the effective maximum c-As optical penetration depth manifesting a Raman signal from the subsurface GaAs core is approximately $25 \mathrm{~nm}$. Formations of c-As exceeding $25 \mathrm{~nm}$ will mask any signal from the interface, and films twice that thickness will effectively prevent the GaAs core from absorbing the above bandgap $(>1.42$ $\mathrm{eV}$ ) share of the terrestrial solar spectrum, a severe limitation on the potential of this approach for PV applications.

\section{Conclusions}

We outline a fabrication process for GaAs nanopillars using low cost self-assembled colloidal nanosphere masks and the MacEtch process. Enhanced PL emission is observed for these GaAs nanostructures, due to increased surface area and reduced emission losses. Observed primary emission peak centered at $1.43 \mathrm{eV}$ and displays a redshift attributable to the presence of $\pi$-GaAs. We observed no indication of any green or blue emission commonly seen in $\pi$-GaAs. From a morphology perspective, improvements to the etch recipe will likely lead to greater homogeneous nanopillar profiles by controlling the lateral etch rate. The formation of the associated GaAs oxides and c-As layer represents a challenge for this technique to many optoelectronic or PV applications.

\section{Acknowledgements}

This work was funded by Enterprise Ireland (CF/2011/1313) under the FlexCell project - Cost-effective Flexible Solar PV system based on the high efficiency III-V compound, Gallium Arsenide. We also acknowledge the Irish Higher Education Authority INSPIRE programme funded by the Irish Government's Programme for Research in Third Level Institutions, Cycle 5, National Development Plan 2007-2013 and the EU/ ERDF/ESF.

\section{References}

1 X. Li, Curr. Opin. Solid State Mater. Sci., 2012, 16, 71-81. 
2 W. Chern, K. Hsu, I. Chun, B. Azeredo, N. Ahmed, K. Kim, J. Zuo, N. Fang, P. Ferreira and X. Li, Nano Lett., 2010, 10, 1582-1588.

3 X. Geng, Z. Qi, M. Li, B. Duan, L. Zhao and P. Bohn, Sol. Energy Mater. Sol. Cells, 2012, 103, 98-107.

4 Z. Huang, N. Geyer, P. Werner, J. de Boor and U. Gösele, Adv. Mater., 2010, 23, 285-308.

5 Y. Yasukawa, H. Asoh and S. Ono, Jpn. J. Appl. Phys., 2010, 49, 116502.

6 H. Asoh, T. Yokoyama and S. Ono, Jpn. J. Appl. Phys., 2010, 49, 046505.

7 M. DeJarld, J. Shin, W. Chern, D. Chanda, K. Balasundaram, J. Rogers and X. Li, Nano Lett., 2011, 11, 5259-5263.

8 Y. Yasukawa, H. Asoh and S. Ono, Electrochem. Commun., 2008, 10, 757-760.

9 X. Zhang, A. Whitney, J. Zhao, E. Hicks and R. Van Duyne, J. Nanosci. Nanotechnol., 2006, 6, 1920-1934.

10 P. Schmuki, J. Electrochem. Soc., 1996, 143, 3316.

11 P. Schmuki, L. Erickson, D. Lockwood, J. Fraser, G. Champion and H. Labbé, Appl. Phys. Lett., 1998, 72, 1039.

12 J. Sabataitye, I. Simkiene and R. A. Bendorius, Mater. Sci. Eng., C, 2002, 19, 155-159.

13 X. Li, Y. Kim, P. Bohn and I. Adesida, Appl. Phys. Lett., 2002, 80, 980.

14 J. Lannin, J. Calleja and M. Cardona, Phys. Rev. B: Solid State, 1975, 12, 585-593.

15 J. Lannin, Phys. Rev. B: Solid State, 1977, 15, 3863-3871.
16 R. Nemanich, G. Lucovsky, W. Pollard and J. Joannopoulos, Solid State Commun., 1978, 26, 137-139.

17 V. Denisov, B. Mavrin and V. Karavanskii, Semiconductors, 2001, 35, 949-952.

18 G. Schwartz, J. Vac. Sci. Technol., 1979, 16, 1383.

19 N. Ali, M. Hashim, A. Abdul Aziz and H. Abu Hassan, Semicond. Sci. Technol., 2008, 23, 055016.

20 T. Onuma, S. Fujioka, T. Yamaguchi, Y. Itoh, M. Higashiwaki, K. Sasaki, T. Masui and T. Honda, J. Cryst. Growth, 2014, 401, 330-333.

21 Z. Sun, L. Yang, X. Shen and Z. Chen, Chin. Sci. Bull., 2012, 57, 565-568.

22 Y. Gao, Y. Bando, T. Sato, Y. Zhang and X. Gao, Appl. Phys. Lett., 2002, 81, 2267.

23 L. Quagliona, Appl. Surf. Sci., 2000, 153, 240-244.

24 C. Thurmond, J. Electrochem. Soc., 1980, 127, 1366.

25 I. Campbell and P. Fauchet, Solid State Commun., 1986, 58, 739-741.

26 H. Richter, Z. Wang and L. Ley, Solid State Commun., 1981, 39, 625-629.

27 M. Watt, C. Torres, H. Arnot and S. Beaumont, Semicond. Sci. Technol., 1990, 5, 285-290.

28 G. Lucovsky and J. Knights, Phys. Rev. B: Solid State, 1974, 10, 4324-4330.

29 G. Schwartz, B. Schwartz, D. DiStefano, G. Gualtieri and J. Griffiths, Appl. Phys. Lett., 1979, 34, 205.

30 H. Richter, Z. Wang and L. Ley, Solid State Commun., 1981, 39, 625-629. 